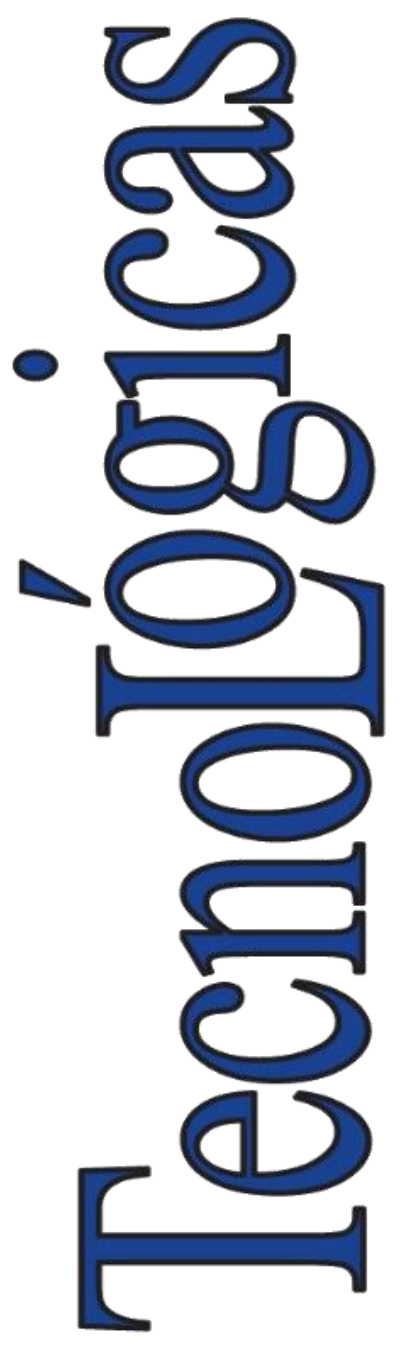

ISSN-p: 0123-7799 ISSN-e: $2256-5337$

Vol. 24, nro. 50, e1686

Recibido: 7 junio 2020 Aceptado: 16 septiembre 2020 Disponible: 11 noviembre 2020

CInstituto Tecnológico Metropolitano Este trabajo está licenciado bajo una Licencia Internacional Creative Commons Atribución (CC BY-NC-SA)

\title{
Evaluación de daños en pavimento flexible usando fotogrametría terrestre y redes neuronales
}

\section{Damage Evaluation in Flexible Pavement Using Terrestrial Photogrammetry and Neural Networks}

\author{
(D) Qizette Tello-Cifuentes ${ }^{1}$; \\ (D) Marcela Aguirre-Sánchez²; \\ iD Jean P. Díaz-Paz; \\ iD Francisco Hernández ${ }^{4}$
}

${ }^{1}$ Universidad del Valle, Cali-Colombia, lizette.tello@correounivalle.edu.co 2Universidad Pontificia Bolivariana, Medellín, Colombia, yurimarcela.aguirre@upb.edu.co ${ }^{3}$ Institución Universitaria Antonio José Camacho, Cali-Colombia, jpdiaz@uniajc.edu.co

${ }^{4}$ Universidad del Valle, Cali-Colombia, francisco.hernandez@correounivalle.edu.co

Cómo citar / How to cite

L. Tello-Cifuentes; M. Aguirre-Sánchez; J. P. Díaz-Paz; F. Hernández, "Evaluación de daños en pavimento flexible usando fotogrametría terrestre y redes neuronales”, TecnoLógicas, vol. 24, nro. 50, e1686, 2021. https://doi.org/10.22430/22565337.1686

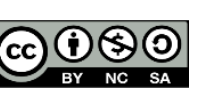




\section{Resumen}

La evaluación del deterioro de las vías en Colombia se realiza por medio de inventarios manuales e inspecciones visuales. Los métodos de evaluación del estado de las vías adoptados por el INVIAS (Instituto Nacional de Vías) son VIZIR (Visión Inspection de Zones et Itinéraires Á Risque) y PCI (Paviment Condition Index). Estos determinan la gravedad de daño en pavimento flexible y rígido; sin embargo, pueden ser tediosos, subjetivos y requieren de la experiencia de un evaluador, lo que evidencia la necesidad de desarrollar metodologías de evaluación del estado de las vías. Este documento presenta una metodología para la evaluación de los deterioros presentes en pavimento flexible usando técnicas de fotogrametría terrestre y redes neuronales que está compuesta por seis etapas: i. Captura de las imágenes, ii. Preprocesamiento de las imágenes, iii. Segmentación mediante técnicas de detección de bordes, iv. Extracción de las características, v. Clasificación utilizando redes neuronales, y vi. Evaluación del área de afectación del deterioro. La metodología se evaluó con imágenes reales de pavimento con tres tipos de deterioro: grieta longitudinal, piel de cocodrilo y bache. Como clasificador se utilizó una red neuronal multicapa con configuración (12 12 3), entrenada utilizando el algoritmo Levenberg Marquardt de retropropagación. Se obtuvo una exactitud del $96 \%$ en el clasificador, una sensibilidad de $93.33 \%$ y una índice kappa de 0.936. Esta metodología es la base para la creación de un sistema automatizado de evaluación del deterioro presente en las vías, el cual puede contribuir en la reducción en tiempo y costo en los planes de gestión de mantenimiento de la infraestructura vial.

\section{Palabras clave}

Fotogrametría terrestre, redes neuronales, grietas en el pavimento, vías terrestres, procesamiento de imágenes.

\section{Abstract}

In Colombia, road deterioration is assessed by means of road inventories and visual inspections. For this assessment, the Instituto Nacional de Vias (Colombia's National Road Institute) (abbreviated INVIAS in Spanish) uses the Vision Inspection de Zones et Itinéraires Á Risque (VIZIR) and Pavement Index Condition (PCI) methods. These two methods serve to determine the severity of damages in flexible and rigid pavements. However, they can be tedious and subjective and require an experienced evaluator, hence the need to develop new methods for road condition assessment. In this paper, we present a methodology to evaluate flexible pavement deterioration using terrestrial photogrammetry techniques and neural networks. The proposed methodology consists of six stages: (i) image capture, (ii) image preprocessing, (iii) segmentation via edge detection techniques, (iv) characteristic extraction, (v) classification using neural networks, and (vi) assessment of deteriorated areas. It is verified using real images of three different pavement distresses: longitudinal cracking, crocodile cracking, and pothole. As classifier, we use a multilayer neural network with a (12 12 3) configuration and trained using the Levenberg-Marquardt algorithm for backpropagation. The results show a classifier's accuracy of $96 \%$, a sensitivity of $93.33 \%$, and a Cohen's Kappa coefficient of $93.67 \%$. Thus, our proposed methodology could pave the way for the development of an automated system to assess road deterioration, which may, in turn, reduce time and costs when designing road infrastructure maintenance plans.

\section{Keywords}

Terrestrial photogrammetry, neural networks, pavement cracking, roadways, image processing. 


\section{INTRODUCCIÓN}

En Colombia, la evaluación del estado del pavimento en las vías se realiza mediante métodos que permiten recopilar y analizar información de estas. El método VIZIR (Visión Inspection de Zones et Itinéraires Á Risque) se utiliza para concreto flexible y permite clasificar los deterioros en degradaciones de tipo A, debidas a insuficiencias en la capacidad estructural, y degradaciones de tipo B, debidas a deficiencias constructivas y a las condiciones locales. Por su parte, el método PCI (Paviment Condition Index) se utiliza para cualquier tipo de pavimento y establece clase, severidad y cantidad de daño presente. Estos métodos se aplican a partir de inventarios manuales e inspecciones visuales [1], [2].

Las técnicas de fotogrametría y procesamiento de imágenes permiten realizar mediciones en sitio, rápido procesamiento de información y bajo costo [3], [4]. De acuerdo con [5], un proceso típico de inspección puede dividirse en las etapas de preprocesamiento, segmentación y clasificación de la imagen. El preprocesamiento se utiliza para mejorar la calidad de la imagen de entrada con el fin de facilitar el análisis y la interpretación en las etapas subsiguientes. La segmentación es utilizada, según [6], para detectar contornos de un objeto y los límites entre el objeto y el fondo de la imagen. La clasificación tiene como principal objetivo determinar la categoría a la que pertenece cada conjunto de características [7].

Los principales métodos de inspección de carreteras conforman tres categorías: manuales, automáticas y por vibraciones. El método Dynaflect opera sobre el principio de una fuerza vibratoria, que se coloca sobre el pavimento por medio de dos pequeñas ruedas de metal [8].

Los métodos manuales o tradicionales, se utiliza un vehículo que viaja por la carretera, se observan imperfecciones del pavimento y se toman apuntes de ubicación y tipo de fisuras. Estos métodos son caros, dispendiosos y poco precisos debido a la subjetividad de la persona que realiza la inspección. En los métodos automáticos se incorpora un vehículo como sensor al realizar adaptaciones de cámaras con el fin de adquirir tomas de la superficie de la carretera [9], [10]. ARAN es un analizador automático de carreteras que utiliza técnicas de procesamiento de imágenes para la identificación de los deterioros en el pavimento y redes neuronales para la clasificación según su tipo: longitudinal, transversal y piel de cocodrilo [11]. PAVUE es un sistema de procesamiento de imágenes en tiempo real para la evaluación automatizada de grietas en el pavimento que utiliza hasta cuatro cámaras de video e iluminación de flash sincronizado para recopilar los datos. Las imágenes de la superficie se analizan mediante un sistema de evaluación de imágenes automatizado (AIES) y, posterior al análisis de los datos, se presentan mapas de grietas con el tipo y la gravedad [12].

Las redes neuronales son una herramienta de clasificación [13] que permite abordar problemas complejos y no lineales. Las redes neuronales son usadas ampliamente en resolver problemas de ingeniería del pavimento como: el diseño y evaluación de mezcla asfáltica, la evaluación de la permeabilidad, la estimación de la tensión plástica y la estimación de las propiedades mecánicas de los materiales de pavimentación [14]. Uno de los tipos de red neuronal más usado es la red neuronal multicapa con algoritmo de aprendizaje Backpropagation (BP) debido a su potencial como herramienta de predicción y su capacidad para extraer información útil de las muestras [11]. En [15] proponen una red neuronal BP con una configuración (2 13 1): 2 neuronas en la capa de entrada, una capa oculta con 13 neuronas y 1 neurona en la capa salida para clasificar grietas en el pavimento. Esta clasificación se realiza posterior a aplicar un procesamiento de imágenes que implica técnicas como: conversión a escala de grises, detectores de bordes y operaciones morfológicas.

Este documento propone una metodología para la evaluación del pavimento flexible usando técnicas de fotogrametría terrestre y redes neuronales; técnicas que han sido utilizadas para resolver problemas en áreas adyacentes por diferentes autores. El artículo se 
ordena de la siguiente manera: la sección 2, muestra los materiales y métodos; la sección 3 , los resultados obtenidos; la sección 4, la discusión de los resultados; y, la sección 5, las conclusiones.

\section{MATERIALES Y MÉTODOS}

La metodología de evaluación propuesta en este documento consta de seis etapas: i. Captura de las imágenes desde un automóvil, ii. Preprocesamiento que permite eliminar la distorsión presente en la imagen y la mejora del contraste, iii. Segmentación mediante técnicas de detección de bordes y la trasformada de Beamlet, iv. Extracción de características, v. Clasificación con redes neuronales artificiales (RNA) y vi. Evaluación del área de afectación (ver Figura. 1).

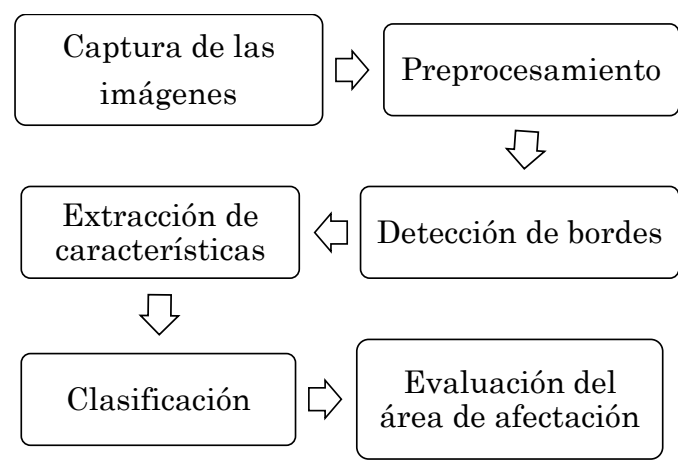

Figura 1. Metodología Propuesta. Fuente: elaboración propia.

\subsection{Captura de las imágenes}

Se desarrolla un montaje experimental que permite recolectar información de forma semiautomática, similar a lo realizado por [12] que utiliza un vehículo al cual le adapta cámaras. El montaje consta de un vehículo, una cámara digital GoPro de 11 Megapíxeles, un celular Samsung S3 y una estructura de soporte para la Figura 2.
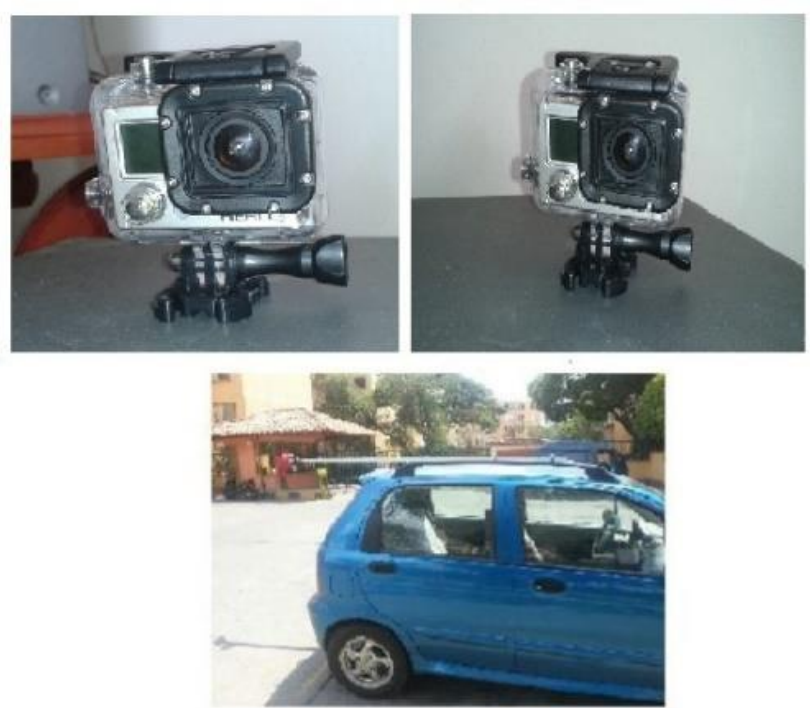

Figura 2. Montaje experimental. Fuente: elaboración propia. 
Para la recolección de la información se capturan imágenes de 2624 x 2880 pixeles a velocidades entre 25 y $30 \mathrm{~km} / \mathrm{h}$, en diferentes horas del día (11am- 1 pm y 3-5pm). Entre estas imágenes se escogen las 365 capturadas a una velocidad de $25 \mathrm{~km}$ entre $3-5 \mathrm{pm}$ debido a que con estas condiciones de captura se obtienen imágenes con menor efecto de borrosidad y sombras. Las 365 imágenes seleccionadas se etiquetan manualmente y se obtienen 228 con el deterioro de tipo grieta longitudinal, 122 del deterioro piel de cocodrilo y 15 del deterioro de tipo bache. Se descartan las imágenes identificadas como defectuosas por cambios repentinos en las condiciones de captura. El dataset seleccionado consta de 30 imágenes con el deterioro grieta longitudinal, 84 con piel de cocodrilo y 15 con baches. A partir de este se conforma un dataset de entrenamiento balanceado con 10 imágenes de cada tipo de deterioro y un dataset de validación con 10 imágenes para los tipos de deterioro grieta longitudinal y piel de cocodrilo y 5 para baches, (ver Tabla 1 ).

Tabla 1 Conjunto de imágenes utilizado en el proceso. Fuente: elaboración propia.

\begin{tabular}{ccccc}
\hline Tipo de Deterioro & Procesamiento & $\begin{array}{c}\text { Extracción de } \\
\text { características }\end{array}$ & Entrenamiento & Validación \\
\hline Grieta longitudinal & 30 & 30 & 10 & 10 \\
Piel de cocodrilo & 84 & 84 & 10 & 10 \\
Bache & 15 & 15 & 10 & 5 \\
\hline
\end{tabular}

\subsection{Preprocesamiento}

Dado que la cámara usada tiene una lente gran angular, se realiza la corrección geométrica de las imágenes mediante el modelo de Zhengyou Zang's [16], se convierten escala de grises y se mejora el contraste utilizando técnicas que emplean funciones de potencia para eliminar los efectos no uniformes de intensidad en el fondo de la imagen [17]. Este proceso requiere convertir la intensidad del fondo en una intensidad constante $B$ elegida de manera heurística. Se divide la imagen en ventanas de tamaños potencias de 2 , en cada ventana se encuentra la media $G_{\text {mean }}$ y los mínimos $G_{\min }$ y máximos $G_{\max }$ de los niveles de gris de la imagen. Se establece un límite superior e inferior para calcular los niveles de gris que se encuentren fuera del rango considerándose ruido, con una limitación de $30 \%$, como lo muestra (1) y (2) [18].

$$
\begin{aligned}
& r_{l}=G_{\text {mean }}-\left(G_{\text {mean }}-G_{\text {min }}\right) * 30 \% \\
& r_{h}=G_{\text {mean }}+\left(G_{\text {max }}-G_{\text {mean }}\right) * 30 \%
\end{aligned}
$$

Para cada ventana se calcula el valor medio $G_{\text {mean }}^{\prime}$ de los niveles de los pixeles que se encuentran dentro del rango comprendido entre $r_{l}$ y $r_{h}$. Finalmente, se modifica la intensidad de la imagen $I$, como se muestra en (3) [18].

$$
I^{\prime}=I \cdot \frac{B}{G_{\text {mean }}^{\prime}}
$$

Donde $B$ es la intensidad de fondo constante. 


\subsection{Detección de bordes}

En las imágenes, el vector gradiente indica las zonas y bordes donde existen cambios de tonalidad [19]. La transformada de Beamlet es un método basado en gradientes que tiene en cuenta la orientación y localización de los objetos lineales o curvos en la imagen, realiza un análisis multiescala de las imágenes y detecta cada segmento de línea mediante una cadena de integrales. La transformada se define como se muestra en (4) [20]-[22].

$$
T_{g}(b)=\int_{b} I(x(l)) d l
$$

Donde $I$ es la imagen y $b$ es el Beamlet (haz elemental). También se puede definir como la suma de valores de los píxeles $I(x, y)$, (ver (5)) [18].

$$
T(b)=\sum(x, y) \in b I(x, y)
$$

La transformada Beamlet ha sido usada con éxito en la detección de líneas, curvas y objetos en imágenes ruidosas provenientes de sensores remotos [21], [23].

El tamaño de ventana que mejor define el deterioro tipo grieta longitudinal es de 16 y el umbral escogido de 0.5, ya que se logra detectar el borde sin perder la forma de este, (ver Figura 3). El umbral escogido para el caso de la piel de cocodrilo y los baches es de 0.7, con un tamaño de ventana de 16. Dado que no se encuentra un umbral en general para los tres tipos de deterioro en la detección de los bordes, las imágenes van a recorrer el mismo proceso con los dos umbrales de 0.5 y 0.7 .

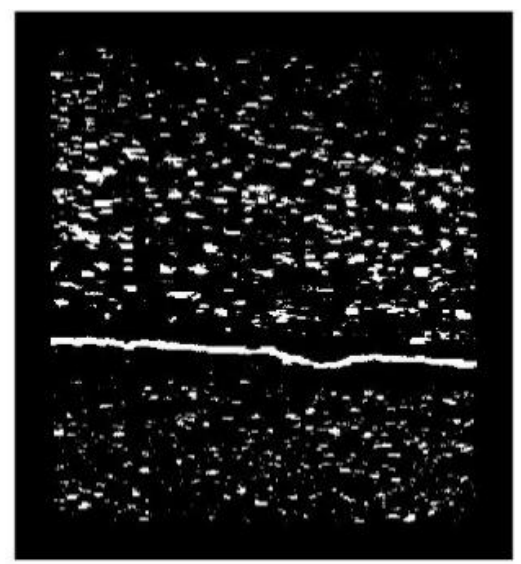

a)

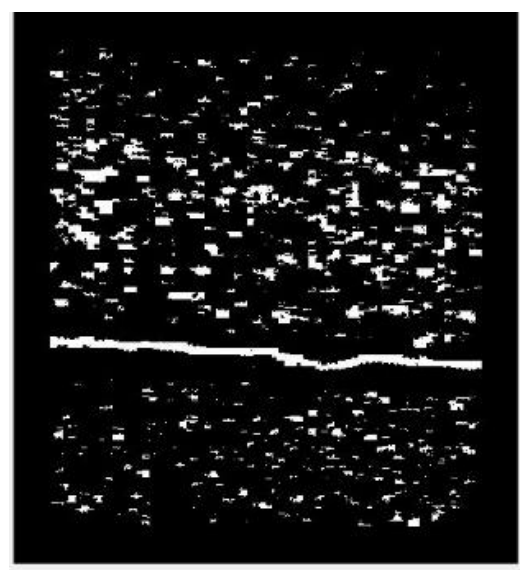

b)

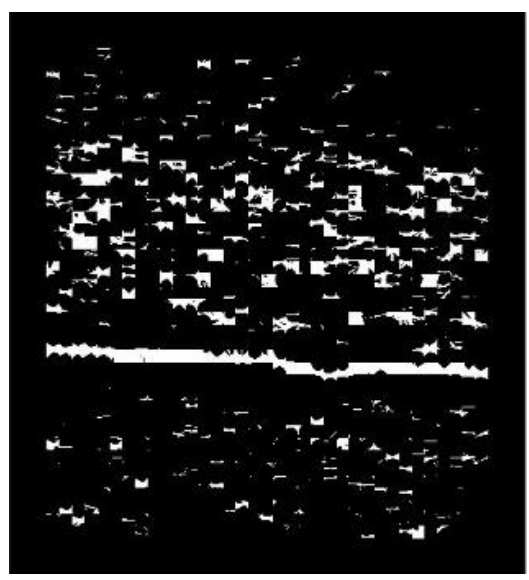

c)

Figura 3. Imagen segmentada a diferentes tamaños de ventana con un umbral de 0.5, a) Con un tamaño de ventana de 16, b) 32 y c) 64 . Fuente: elaboración propia.

Las operaciones morfológicas permiten modificar la forma de los objetos en una imagen a partir de cambios en la intensidad de los pixeles en una vecindad [24]. Las operaciones morfológicas básicas son la erosión y la dilatación [19]. La erosión es aplicada para eliminar el ruido presente en la imagen y la dilatación para rellenar espacios vacíos. Se aplica matemática morfológica con el fin de eliminar el ruido presente en la imagen y extraer el deterioro y las posibles conexiones entre ellos. La Figura 4 ilustra el proceso para las imágenes segmentadas. 


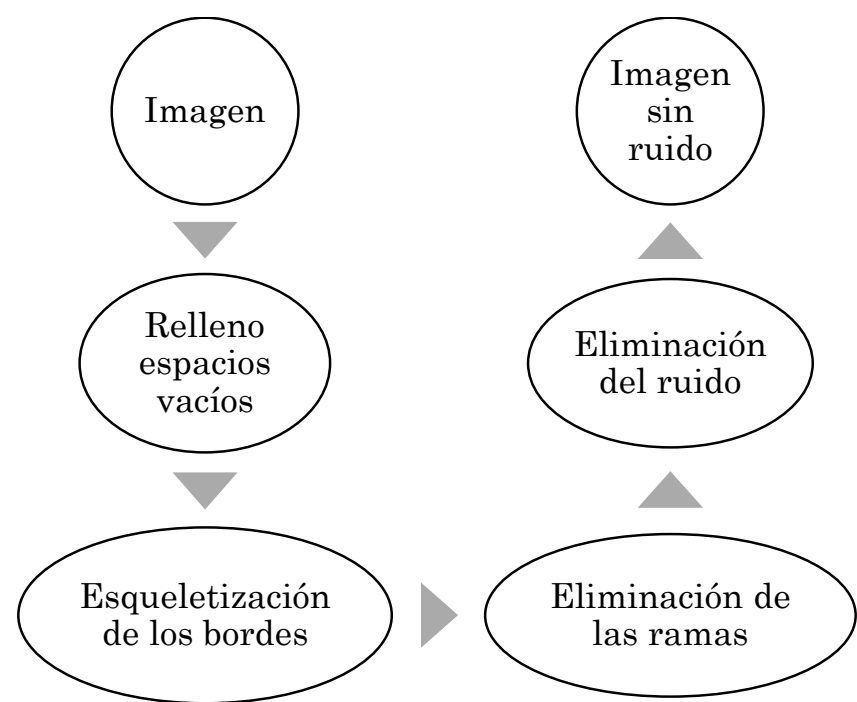

Figura 4. Diagrama de flujo de las operaciones morfológicas utilizadas. Fuente: elaboración propia.

Se rellenan los espacios que quedan vacíos después de la umbralización (ver Figura 5), y se esqueletizan los bordes con el fin de detectar el eje medio del borde. Sin embargo, al realizar esta operación morfológica, se generan una serie de ramificaciones no deseadas en el eje del borde que deben ser eliminadas para dejar solamente el deterioro [24].

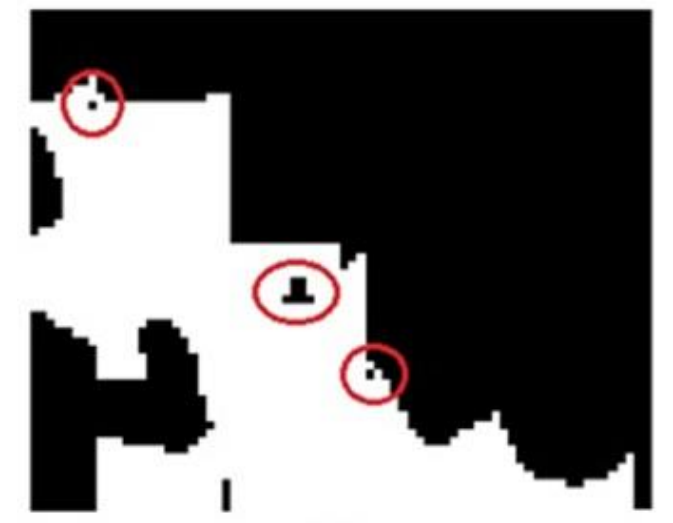

a)

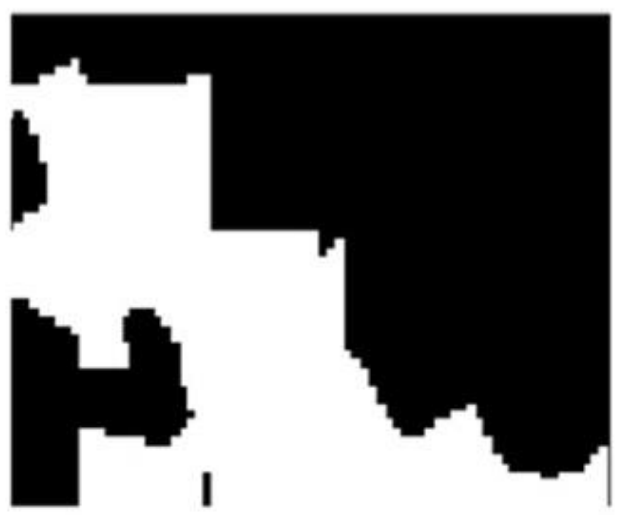

b)

Figura 5. a) Sección de la grieta posterior a la transformada de Beamlet y b) sección después de relleno de espacios vacíos. Fuente: elaboración propia.

En el caso de la grieta longitudinal, se tiene en cuenta la orientación como criterio para su identificación. Esta es obtenida a través del etiquetado de cada región de la imagen [25]. A partir del proceso de segmentación se obtienen dos imágenes. Estas imágenes se someten a una inspección a distintos umbrales, con el fin de conocer el umbral que mejor elimine el ruido.

Debido a que no se logra encontrar un solo umbral, es necesario aplicar diferentes umbrales según el tipo de deterioro (ver Tabla 2), generando tres imágenes. A estas imágenes se les calcula el área de los objetos y se define un umbral de forma heurística, en donde si el área es menor a los 1000 pixeles se considera pavimento sano y la imagen sale del proceso. 
Tabla 2. Umbrales escogidos en la matemática morfológica. Fuente: elaboración propia.

\begin{tabular}{cccc}
\hline Proceso & Grieta longitudinal & Bache & Piel de cocodrilo \\
\hline Eliminar ramas & 200 & 50 & 75 \\
Eliminar el ruido & 350 & 450 & 350 \\
\hline
\end{tabular}

\subsection{Extracción de características}

La extracción de características se utiliza para encontrar la información relevante que permita discriminar a qué clase pertenece un elemento. Un vector de características debe cumplir con 5 propiedades importantes: discriminación, fiabilidad, incorrelación, cálculo y dimensionalidad [25]. Los momentos invariantes pueden considerarse como un promedio ponderado de los pixeles de la imagen y se basan en los momentos geométricos que son invariantes al cambio de escala, traslación y rotación [26]. Los momentos invariantes son calculados sobre la información del contorno de la forma y la región interior de los objetos presentes en la imagen [27].

El vector de características definido para la metodología se forma al concatenar los cuatro primeros momentos invariantes de las tres imágenes resultantes de la etapa de detección de bordes. Así, el vector de características para la imagen es de 12 dimensiones.

\subsection{Clasificación}

Se usa un clasificador supervisado basado en redes neuronales artificiales tipo perceptrón multicapa (MLP) [14]. Para esto se utiliza el Toolbox de Matlab Neural Network, para una red neuronal MLP con algoritmo de aprendizaje Backpropagation Levenberg-Mardquardt.

El número de neuronas en la capa oculta se determinó de forma experimental [28].

\subsection{Evaluación del deterioro}

Para evaluar el deterioro de las vías se adaptó el manual de deterioros de pavimentos flexible [29] para los tres tipos de deterioro: piel de cocodrilo, grietas longitudinales y baches.

\section{RESULTADOS}

Para evaluar el desempeño de la metodología propuesta se realizó una captura de imágenes en pavimentos reales, se etiquetaron las imágenes manualmente. Además, se determinó la mejor estructura para una red MLP en la etapa de clasificación y se evaluó un conjunto de imágenes usando una adaptación del manual de deterioros de pavimento flexible.

La metodología presentó un tiempo de procesamiento de aproximadamente 9 minutos por imagen. A continuación, se describen las pruebas realizadas.

\subsection{Clasificación usando RNA}

Se construyeron 15 RNA con 12 neuronas en la capa de entrada. El vector de entrada está compuesto por los 4 primeros momentos invariantes de las tres imágenes resultantes de la etapa de detección de bordes; la capa de salida está compuesta por 3 neuronas que codifican el tipo de deterioro, como se muestra en la Tabla 3. 
Tabla 3. Codificación de los tipos de deterioros. Fuente: elaboración propia.

\begin{tabular}{cc}
\hline Tipo de Deterioro & Codificación \\
\hline Bache & 100 \\
Piel de cocodrilo & 010 \\
Grieta Longitudinal & 001 \\
\hline
\end{tabular}

Debido a que solo se capturaron 15 deterioros de tipo bache y esto es un limitante en la cantidad de patrones a emplear de las demás clases, ya que en este proceso se debe tener en cuenta el máximo número de imágenes o patrones de cada clase para que la RNA no reconozca más un patrón que otro, se emplearon en el proceso de entrenamiento 30 imágenes, 10 por cada deterioro.

Para determinar el mejor clasificador basado en una red MLP, se entrenaron distintas redes variando la cantidad de neuronas en la capa oculta entre 1 y 15 , entrenando con el dataset balanceado de 10 imágenes de cada deterioro y se escogieron las 6 redes de menor error medio cuadrático (ver Tabla 4).

Tabla 4. Resultados del clasificador con diferentes neuronas en la capa oculta. Fuente: elaboración propia.

\begin{tabular}{cccccc}
\hline $\begin{array}{c}\text { N- de neuronas en la } \\
\text { capa oculta }\end{array}$ & $\begin{array}{c}\text { Error medio } \\
\text { cuadrático }\end{array}$ & Exactitud (\%) & Sensibilidad (\%) & $\begin{array}{c}\text { Precisión } \\
\text { (\%) }\end{array}$ & Kappa \\
\hline 3 & 0.045 & 60.00 & 66.66 & 44.44 & 0.444 \\
5 & 0.044 & 72.00 & 67.66 & 64.33 & 0.567 \\
6 & 0.017 & 88.00 & 80.00 & 92.30 & 0.805 \\
7 & 0.040 & 84.00 & 76.67 & 90.48 & 0.740 \\
8 & 0.039 & 88.00 & 80.00 & 92.30 & 0.805 \\
12 & 0.038 & 96.00 & 93.33 & 96.97 & 0.936 \\
\hline
\end{tabular}

La red neuronal escogida presenta la siguiente configuración (12 12 3). A pesar de no ser la de menor error medio cuadrático obtuvo una mayor exactitud en la clasificación (ver Tabla 4). Esta presentó el mejor desempeño entre los clasificadores evaluados con una exactitud global del 96 \%, una precisión de $96.97 \%$, una sensibilidad de $93.33 \%$ y un índice kappa de 0.936 .

La matriz de confusión obtenida para la RNA (12 12 3) se muestra en la Tabla 5. Esta confundió únicamente un deterioro de tipo bache con piel de cocodrilo. Teniendo en cuenta que algunas de las imágenes escogidas para la validación no se encontraban totalmente libres de ruido y presentaban el deterioro más de una vez, se trató de encontrar la red neuronal que mejor clasificara a pesar de estos problemas.

Tabla 5. Matriz de confusión para la red neuronal con configuración (12 12 3). Fuente: elaboración propia.

\begin{tabular}{cccc}
\hline Deterioro & Bache & Piel de cocodrilo & Longitudinal \\
\hline Bache & 4 & 1 & 0 \\
Piel de cocodrilo & 0 & 10 & 0 \\
Longitudinal & 0 & 0 & 10 \\
\hline
\end{tabular}

\subsection{Evaluación del nivel de afectación}

Se evaluó el nivel de afectación para cada una de las imágenes. Para el caso del deterioro tipo bache (ver Figura 6), la imagen A obtuvo un área de afectación del $6 \%$, lo que indica un 
grado de afectación medio; para el deterioro piel de cocodrilo imagen $\mathrm{B}$, el área de afectación fue de aproximadamente el $12 \%$, lo cual muestra un grado de afectación de nivel medio; y, para la grieta longitudinal imagen $\mathrm{C}$, el grado de afectación fue de $1.45 \%$, equivalente a nivel ligero. El cálculo se realizó considerando que el área del deterioro se encuentra en función del número de pixeles, por lo que el grado de afectación estará influenciado por ellos.

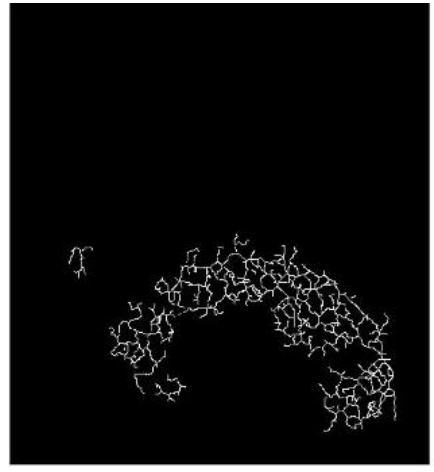

a)

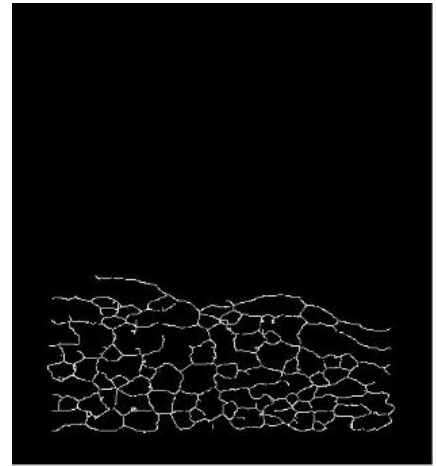

b)

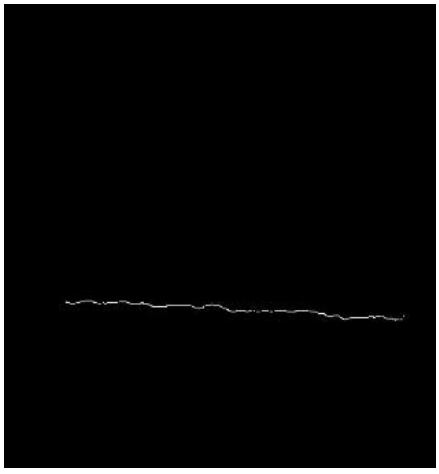

c)

Figura 6. Imágenes de prueba para cada uno de los deterioros, a) bache, b) piel de Cocodrilo y c) grieta longitudinal. Fuente: elaboración propia.

\section{DISCUSIÓN}

Como clasificador de los deterioros se utilizó una red neuronal perceptrón multicapa con configuración (12 12 3). El clasificador tuvo una exactitud general del $96 \%$, producto de confundir un deterioro tipo bache con el deterioro piel de cocodrilo. Esta arquitectura del clasificador es similar a la utilizada por [13], que utilizó una red neuronal con configuración (3 8 3) y obtuvo una exactitud del $97.5 \%$. Sin embargo, [13] no considera en su clasificación el deterioro tipo piel de cocodrilo. Por otro lado, [3] utilizó una red neuronal MLP con configuración (2 34 ) y obtuvo una exactitud del clasificador $98.6 \%$, pero no incorporó el deterioro tipo bache. La Tabla 6 muestra las exactitudes obtenidas por diferentes autores en cuanto a la clasificación de deterioros en el pavimento.

Tabla 6. Resultados obtenidos en este estudio y por diferentes autores. Fuente: elaboración propia.

\begin{tabular}{cccccccc}
\hline & \multicolumn{5}{c}{ Exactitud del clasificador (\%) } \\
\cline { 2 - 7 } Estudio & Total & $\begin{array}{c}\text { Grieta } \\
\text { longitudinal }\end{array}$ & $\begin{array}{c}\text { Grieta } \\
\text { transversal }\end{array}$ & $\begin{array}{c}\text { Grieta } \\
\text { bloque }\end{array}$ & $\begin{array}{c}\text { Piel de } \\
\text { cocodrilo }\end{array}$ & Bache & $\begin{array}{c}\text { Sin } \\
\text { deterioro }\end{array}$ \\
{$[13]$} & 97.5 & 97.5 & 100 & - & - & 95.0 & - \\
{$[11]$} & 92.5 & 88.0 & 100 & - & 97.5 & - & - \\
{$[30]$} & 84.2 & 89.5 & 82.0 & - & 77.5 & - & 88.0 \\
{$[3]$} & 98.6 & 98.4 & 98.4 & 97.6 & 100 & - & - \\
Propio & 96.0 & 100 & - & - & 100 & 80 & - \\
\hline
\end{tabular}

La transformada de Beamlet, a comparación de los operadores clásicos, ofrece una representación óptima y mayor precisión de la posición, localización y orientación en la detección del borde; sin embargo, es necesario aplicar una etapa de limpieza de bordes para evitar falsas detecciones de grieta, como lo menciona [5], [20]. Al igual que en el trabajo de [23], se presentaron problemas en la detección de las fisuras en el pavimento debido a que se 
genera ruido y falla en los puntos de conexión lineales, aunque se preserva una cantidad significativa de los deterioros y se observa, al igual que [21], que el procesamiento por medio de este método inhibe la subjetividad visual humana y da lugar a una serie de haces de líneas con orientación, longitud y ubicación precisa de las grietas presentes en el pavimento.

El tiempo de procesamiento en este proyecto es de aproximadamente 9 minutos para una imagen de 2624 x 2880 pixeles. Esta medición está influenciada por las características del hardware utilizado. La transformada de Beamlet es el proceso de la metodología propuesta que demanda más recursos computacionales, lo que aumenta considerablemente el tiempo de procesamiento reportado en [23], que indica que el tiempo de procesamiento en su proyecto oscila entre 18.23 y 34.72 segundos para una imagen de 256 x 256 pixeles.

A diferencia de [7], que utilizó en su estudio los siete momentos invariantes, y de [26], que empleó una mejora de los momentos invariantes de $\mathrm{Hu}$ agregando un octavo momento, en este proyecto se utilizaron los primeros cuatro momentos invariantes como vector de características, obteniendo un porcentaje de aciertos del $96 \%$.

\section{CONCLUSIONES}

Se desarrolló una metodología semiautomática que permite la detección de distintos deterioros en pavimentos (grieta longitudinal, bache y piel de cocodrilo) y realiza una evaluación cuantitativa de los mismos, lo que la convierte en insumo para la oportuna toma de decisiones en cuanto al mantenimiento de la malla vial.

Entre los clasificadores evaluados, la arquitectura con mejor desempeño para la clasificación de los deterioros presentes en el pavimento flexible es una red neuronal perceptrón multicapa con configuración (12 12 3), entrenada con el algoritmo Levenberg Marquardt de retro propagación. Este clasificador obtuvo un porcentaje de aciertos del $96 \%$, una precisión total del $96.97 \%$, una sensibilidad de 93.33 \% y una índice kappa de 0.936. Para los tipos de deterioro piel de cocodrilo y grieta longitudinal se alcanzó una exactitud en la clasificación del 100 \% y 80 \% para el deterioro tipo bache.

El sistema propuesto para la evaluación del deterioro de las vías puede complementarse incorporando sistemas de iluminación que disminuyan el efecto de sombras en las imágenes e información de la ubicación espacial de los deterioros detectados. Además, se recomienda introducir clasificadores basados en redes convolucionales que pueden mejorar la precisión de la detección al usar mayores recursos computacionales y algoritmos más elaborados.

Finalmente, se recomienda adaptar y aplicar esta metodología para abarcar los tipos de pavimento flexible y rígido.

\section{AGRADECIMIENTOS}

Universidad del Valle por el apoyo para el desarrollo de la investigación. El artículo no cuenta con ninguna financiación o apoyo económico.

\section{CONFLICTOS DE INTERÉS DE LOS AUTORES}

Los autores declaran que no se presentan conflictos de interés. 


\section{CONTRIBUCIÓN DE LOS AUTORES}

Lizette Tello-Cifuentes autor de conceptualización, diseño y desarrollo de la investigación, para tesis de pregrado y escritora del artículo.

Marcela Aguirre- S. autor de conceptualización, diseño y desarrollo de la investigación, para tesis de pregrado y apoyo en la escritura del artículo.

Jean P. Díaz-Paz autor que contribuyó como director de tesis, durante el planteamiento y desarrollo de la investigación y apoyo en la escritura del artículo.

Francisco Hernández autor que contribuyó como director de tesis, durante el planteamiento y desarrollo de la investigación y apoyo en la escritura del artículo.

\section{REFERENCIAS}

[1] L. F. Macea; L. Morales; L. G. Márquez, "Un sistema de gestión de pavimentos basado en nuevas tecnologías para países en vía de desarrollo," Ing. Investig. Tecnol., vol. 17, no. 2, pp. 223-236, Apr. 2016. https://doi.org/10.1016/j.riit.2016.06.007

[2] V. G. Cerón, "Evaluación y comparación de metodologías VIZIR y PCI sobre el tramo de vía en pavimento flexible y rígido de la vía: Museo Quimbaya-CRQ Armenia Quindío (PR 00+000-PR 02+600)," (trabajo de especialización) Facultad de ingeniería y arquitectura, Universidad Nacional de Colombia. Manizales. 2006. URL

[3] H. Rababaah; D. Vrajitoru; J. Wolfer, "Asphalt Pavement Crack Classification: A Comparative Study of Three AI Approaches: Multilayer Perceptron, Genetic Algorithms and Self-Organizing Maps," (Tesis de Mestría), Indiana University South Bend, 2005. URL

[4] J. Valença; E. Júlio; H. Araújo, "Aplicações De Fotogrametria Na Monitorização De Estruturas,” in 2007 ICM In National Conference on A Instrumentação Científica e a Metrologia Aplicadas à Engenharia Civil, Lisboa 2007. pp. 2- 9. URL

[5] Y. Sun; E. Salari; E. Chou, "Automated pavement distress detection using advanced image processing techniques," in 2009 IEEE International Conference on Electro/Information Technology, Windsor. 2009, pp. 373-377. http://doi.org/10.1109/EIT.2009.5189645

[6] S. Alamri, N. Kalyankar; K. S. D., "Image Segmentation by Using Edge Detection," IJCSE Int. J. Comput. Sci. Eng., vol. 2, no. 3, 2010, pp. 804-807. URL

[7] H. Mu; D. Qi, "Pattern Recognition of Wood Defects Types Based on Hu Invariant Moments," in 2009 2nd International Congress on Image and Signal Processing, Tianjin. 2009, pp. 1-5. https://doi.org/10.1109/CISP.2009.5303866

[8] T. C. Ling, "Evaluation of cracks and disintegrations using close range digital photogrammetry and image processing technique," (Tesis de Maestría), Faculty of Civil Engineering, Universiti Teknologi Malaysia, 2005. URL

[9] A. Ragnoli; M. R. De Blasiis; A. Di Benedetto, "Pavement Distress Detection Methods: A Review," Infrastructures, vol. 3, no. 4, p. 58, Dec. 2018. https://doi.org/10.3390/infrastructures3040058

[10] S. Wu; J. Fang; X. Zheng; X. Li, "Sample and Structure-Guided Network for Road Crack Detection,” IEEE Access, vol. 7, pp. 130032-130043, Sep. 2019. https://doi.org/10.1109/ACCESS.2019.2940767

[11] L. Li; L. Sun; G. Ning; S. Tan, "Automatic Pavement Crack Recognition Based on BP Neural Network," Promet Traffic Transportation, vol. 26, no. 1, pp. 11-22, Feb. 2014. https://doi.org/10.7307/ptt.v26i1.1477

[12] M. Gavilán et al., "Adaptive Road Crack Detection System by Pavement Classification,” Sensors, vol. 11, no. 10, pp. 9628-9657, Oct. 2011. https://doi.org/10.3390/s111009628

[13] A. Banharnsakun, "Hybrid ABC-ANN for pavement surface distress detection and classification," Int. J. Mach. Learn. Cybern., vol. 8, no. 2, pp. 699-710, Apr. 2017. https://doi.org/10.1007/s13042-015-0471-1

[14] H. Gong; Y. Sun; W. Hu; B. Huang, "Neural networks for fatigue cracking prediction using outputs from pavement mechanistic-empirical design," Int. J. Pavement Eng., pp. 1-11, Feb. 2019. https://doi.org/10.1080/10298436.2019.1580367

[15] G. K. Choudhary; S. Dey, "Crack detection in concrete surfaces using image processing, fuzzy logic, and neural networks," in 2012 IEEE Fifth International Conference on Advanced Computational Intelligence (ICACI), China. 2012, pp. 404-411. https://doi.org/10.1109/ICACI.2012.6463195

[16] M. Qi; B. Zhang; Y. Xu; H. Xin; G. Cheng, "Linear Camera Calibration by Single Image Based on Distortion Correction," in Proceedings of the 2nd International Conference on Graphics and Signal Processing, Sydney. 2018, pp. 21-25. https://doi.org/10.1145/3282286.3282303 
[17] E. Rivas; J. Mendiola; G. Herrera; C. Gonzáles; M. Trejo; G. Ríos, "Mejora de Contraste y Compensación en Cambios de la Iluminación," Comput. Sist., vol. 10, no. 4, pp. 357-371, 2007. URL

[18] L. Ying; E. Salari, "Beamlet Transform-Based Technique for Pavement Crack Detection and Classification," Comput. Aided Civ. Infrastruct. Eng., vol. 25, no. 8, pp. 572-580, Jun. 2010.

https://doi.org/10.1111/j.1467-8667.2010.00674.x

[19] M. Carrasco Zambrano, "Segmentación de Fallas en Soldaduras Utilizando Técnicas de Procesamiento Digital de Imágenes," (Tesis de Maestría), Universidad de Santiago De Chile, Chile, 2003. URL

[20] L. Ling; H. Peikang; W. Xiaohu; P. Xudong, "Image edge detection based on beamlet transform," J. Syst. Eng. Electron., vol. 20, no. 1, pp. 1-5, Feb. 2009. URL

[21] N. Wei; X. Zhao; X. Y. Dou; H. Song; T. Wang, "Beamlet Transform Based Pavement Image Crack Detection," in 2010 International Conference on Intelligent Computation Technology and Automation, Changsha. 2010, pp. 881-883. https://doi.org/10.1109/ICICTA.2010.755

[22] A. Ouyang; Y. Wang, "Edge Detection in Pavement Crack Image with Beamlet Transform," in 2nd International Conference on Electronic \& Mechanical Engineering and In-formation Technology, Sep. 2012, pp 2036-2039. https://doi.org/10.2991/emeit.2012.451

[23] Z. Lin; X. Zhao, "Geometrical flow-guided fast beamlet transform for crack detection," IET Image Process., vol. 12, no. 3, pp. 382-388, Nov. 2018. https://doi.org/10.1049/iet-ipr.2017.0747

[24] A. Cubero-Fernandez; F. J. Rodriguez-Lozano; R. Villatoro; J. Olivares; J. M. Palomares, "Efficient pavement crack detection and classification," EURASIP J. Image Video Process., vol. 2017, no. 39, pp. 111, Dec. 2017. https://doi.org/10.1186/s13640-017-0187-0

[25] E. A. Sobrado Malpartida, "Sistema de visión artificial para el reconocimiento y manipulación de objetos utilizando un brazo robot," (Tesis de Maestría), Pontificia Universidad Católica del Perú, Lima, 2003. URL

[26] M. E. G. Mital; H. V. Villaruel; E. P. Dadios, "Neural Network Implementation of Divers Sign Language Recognition based on Eight Hu-Moment Parameters," in 2018 2nd International Conference on Informatics and Computational Sciences (ICICoS), $\quad$ Semarang. $2018, \quad$ pp. https://doi.org/10.1109/ICICOS.2018.8621642

[27] J. Zhao; X. Wang, "Vehicle-logo recognition based on modified HU invariant moments and SVM," Multimed. Tools Appl., vol. 78, no. 1, pp. 75-97, Oct. 2017. https://doi.org/10.1007/s11042-017-5254-0

[28] H. S. Yoo; Y. S. Kim, "Development of a crack recognition algorithm from non-routed pavement images using artificial neural net-work and binary logistic regression," KSCE J. Civ. Eng., vol. 20, no. 4, pp. 11511162, May 2016. https://doi.org/10.1007/s12205-015-1645-9

[29] Universidad Nacional sede Bogotá; Ministerio de transporte Instituto Nacional de Vías INVIAS, "Estudio e investigación del estado actual de las obras de la red nacional de carreteras Convenio Interadministrativos 0587-03. Manual para la inspección visual de Pavimentos Flexibles.” Bogotá, octubre 2006. URL

[30] N.-D. Hoang; Q.-L. Nguyen, "A novel method for asphalt pavement crack classification based on image processing and machine learning," Eng. Comput., vol. 35, no. 2, pp. 487-498, Apr. 2019. https://doi.org/10.1007/s00366-018-0611-9 